\title{
Mercaderes en el Templo: hegemonía del paradigma bio-comercial en Psiquiatría.
}

\author{
Money Changers in the Temple: The bio-commercial paradigm hegemony in nowadays \\ Psychiatry.
}

\author{
Jose García-Valdecasas Campeloa, Amaia Vispe Astolab
}

${ }^{a}$ Psiquiatra. Hospital Universitario de Canarias. Servicio Canario de la Salud. ${ }^{b}$ Enfermera Especialista en Salud Mental. Hospital Universitario Nuestra Señora de La Candelaria. Servicio Canario de la Salud.

Correspondencia: Jose García-Valdecasas Campelo (jose_valdecasas@hotmail.com)

Recibido: 21/09/2010; aceptado: 03/12/2010

\begin{abstract}
RESUMEN: En el presente trabajo nos proponemos llevar a cabo una reflexión sobre la influencia de la industria farmacéutica en la psiquiatría actual, a través de una revisión de cuatro aspectos: la formación docente y científica que damos y recibimos, así como sus sesgos; la nosografía psiquiátrica contemporánea y sus próximas evoluciones según anuncia ya el DSM-V; la eficacia y efectos secundarios de los fármacos que prescribimos, medidos a través de estudios no dependientes de las empresas que venden dichos productos; $y$, en último lugar, el papel que juega el gasto farmacéutico que provocamos y si se justifica o no, teniendo en cuenta la coyuntura de crisis económica global en la que estamos inmersos. Finalmente, elaboraremos unas conclusiones en base a lo revisado y a nuestra propia experiencia personal en la relación de nuestra disciplina con la industria. Apuntaremos, humildemente, algunas posibles soluciones para lo que consideramos una situación problemática de la psiquiatría.

PALABRAS CLAVE: Industria farmacéutica, medicina basada en evidencia, nosografía psiquiátrica, formación psiquiátrica, gasto farmacéutico.
\end{abstract}

\begin{abstract}
With this paper we pursue a careful consideration about the role and the influence of the pharmaceutical industry on nowadays psychiatry by reviewing and analyzing four capital aspects: the medical and scientific education we receive and provide, as well as its bias; the contemporary psychiatric nosography and its probable evolution considering the oncoming DSM-V; the effectiveness and side effects of the drugs we prescribe, measured by independent studies not provided by the pharmaceutical companies that sell these drugs; and last but not least, the pharmaceutical expenditure we cause with our prescriptions, trying to elucidate whether this expenditure is justified considering the global economic crisis we are living. Finally, we summarize our review and our professional and personal experience in some conclusions to define the relationship between the pharmaceutical industry and the practice of psychiatry, providing, from our humble point of view, some solutions for what we consider a problematic situation of psychiatry.

KEY WORDS: Pharmaceutical industry, evidencebased medicine, psychiatric nosography, psychiatric education, pharmaceutical expenditure.
\end{abstract}

\section{Introducción}

La principal semejanza entre el DSM y la Biblia es que, en nuestro entorno histórico y sociocultural, aunque uno se defina como ateo en tales dogmas, no por ello dejan de hacer sentir su influencia en nuestra forma de ver el mundo y comportarnos en él. Ya sea en la vida o en la profesión. Esta introducción viene a propósito del título que hemos escogido para nuestro trabajo. En cita del Nuevo Testamento, Jesucristo entró en el Templo de Jerusalén y la emprendió a golpes con los merca- 
deres que allí se encontraban al grito de "¡Habéis convertido la casa de mi Padre en una cueva de ladrones!". Y nos parece obvio que Jesús se equivocó. Al fin y al cabo, los mercaderes a nadie engañaban, ofreciendo sus productos abiertamente y sin que hubiera dudas acerca de su intención lucrativa. ¿No hubiera sido más apropiado responsabilizar de tal situación a los sacerdotes encargados de guardar el Templo? Un poco de esto trata nuestro estudio.

En nuestra búsqueda, han sido varias las publicaciones encontradas que en diversos medios se han preguntado acerca del modo de actuación de la industria farmacéutica y sus relaciones con la psiquiatría como institución y disciplina. Ya Mata y Ortiz en un artículo imprescindible (1), señalaban que en las últimas décadas se había asistido al desarrollo de una psiquiatría al servicio del mercado: una psiquiatría no biológica sino comercial. Por nuestra parte y siguiendo en esa línea, hablamos en un trabajo previo (2) de la supuesta hegemonía del llamado paradigma biológico en psiquiatría, para resaltar también su carácter esencialmente comercial. Es lo que hemos venido a denominar en nuestro título paradigma bio-comercial, por supuesto no en el sentido de Kuhn como aquel conjunto de teorías capaz de dar una explicación coherente a una determinada rama del conocimiento, sino por desgracia más bien como ese conjunto de prácticas muchas veces no del todo conscientes que configuran una forma de trabajar tal vez más influida por la industria farmacéutica de lo que creemos o querríamos.

Consideramos que dicha influencia se podría analizar a través de cuatro apartados diferentes. Por supuesto, tal división es un tanto artificial pues, como veremos, estos cuatro apartados se solapan ampliamente, pero pueden ser útiles a la hora de ordenar nuestra exposición. En primer lugar, estudiaremos la influencia de la industria farmacéutica sobre el propio bagaje científico de nuestra disciplina, nuestra formación continuada y la que proporcionamos a nuestros residentes, tanto desde un punto de vista teórico como práctico. En segundo lugar y en estrecha relación con el anterior, analizaremos cómo intereses mercantiles tienen mucho que decir y ganar en la construcción de nuestras clasificaciones y nosografías. En tercer lugar, prestaremos atención a distintos estudios sobre psicofármacos, no financiados por la industria ni presentados por nuestros visitadores médicos habituales, que cuestionan las consideraciones de eficacia y seguridad que nos son repetidas machaconamente desde dichos ámbitos. En cuarto y último lugar, creemos imprescindible una reflexión acerca del gasto sanitario que generamos como prescriptores y su justificación en términos de resultados clínicos, y más aún en los tiempos que corren de crisis global y de peligro evidente para la supervivencia del estado de bienestar tal y como lo hemos conocido y disfrutado hasta ahora. 
ORIGINALES Y REVISIONES

Industria farmacéutica, ciencia y formación.

Siguiendo nuevamente a Mata y Ortiz, es evidente la importancia que para la industria farmacéutica tiene el mantenimiento de un modelo biomédico de la enfermedad mental en que sus productos sean siempre la parte imprescindible de la terapia. La industria presume de hacer un gasto importante en investigación y desarrollo, pero es conocido que una parte sustancial de dichos recursos se dedica a ensayos clínicos que no son otra cosa que campañas de marketing apenas disimuladas. Estos ensayos clínicos, remunerados por supuesto, metodológicamente pobres y de dudosa ejecución son esgrimidos luego para justificar supuestas eficacias y tolerancias, aparte de lograr inducir la prescripción de un determinado producto. Gran parte de la formación continuada que la industria realiza con los profesionales, en congresos, charlas o reuniones científicas de diversa índole se basa en la promoción de sus productos directa o indirectamente, previo pago al ponente de turno. Como señaló un artículo del British Medical Journal de 2002 (3), parece demostrado que la investigación financiada directa o indirectamente por la industria tiene un sesgo sistemático que la favorece.

La preocupación en el seno de revistas de prestigio tales como el British Medical Journal y The Lancet por la influencia de los intereses económicos en la calidad de la información científica de los artículos, llevó en 2001 a un editorial conjunto denunciando la situación (4,5). Como recoge The Lancet (6), las reglas del Comité Internacional de Editores de Publicaciones Médicas establecen que quienes toman las decisiones en publicaciones de este tipo no deben tener intereses personales, profesionales o financieros en los temas que tratan. Sin embargo es frecuente ver, sobre todo en las revistas de ámbito nacional aunque no exclusivamente, en los consejos editoriales los mismos nombres que dirigen investigaciones financiadas por la industria y que además dan los cursos y seminarios que patrocinan un determinado producto.

Un fenómeno en alza es el llamado "ghostwriting", técnica por la cual los artículos encargados por una determinada empresa son firmados por autores de prestigio que no han participado directamente en el análisis de los datos (7). En algunos casos, una compañía farmacéutica paga a una empresa por la redacción de un artículo acerca de una investigación con resultados acordes con las necesidades de la compañía, y entonces, se busca un nombre de prestigio a quien se le propone ser el autor (8).

Es conocido también el sesgo de publicación (9) por el que estudios con resultados positivos se publican en mayor medida mientras que aquellos con resultados nulos o negativos muchas veces no llegan a las revistas científicas.

Un interesante trabajo publicado en el New England Journal of Medicine (10) encuentra que, de 74 estudios registrados por la FDA americana acerca de la 
eficacia de distintos antidepresivos, el $31 \%$ (incluyendo 3.449 participantes) no fueron publicados. Se publicaron 37 estudios con resultados positivos para el fármaco analizado y sólo uno con resultado positivo no se publicó. Por el contrario, de los estudios con resultados negativos o cuestionables, 3 se publicaron, 22 no fueron publicados y 11 se publicaron de tal manera que, en opinión de Turner et al, inducían a percibir un resultado positivo. En la literatura publicada, el 94\% de los ensayos fueron positivos, mientras que los análisis de la FDA mostraban sólo un 51\% de resultados positivos. Metaanálisis separados de los datos de la FDA y de las publicaciones muestran que el incremento en el tamaño del efecto oscila entre un $11 \%$ y un $69 \%$ para cada fármaco individualmente, siendo del $32 \%$ para el total.

Por ello, la información de las revistas científicas se convierte en no representativa de la totalidad de la evidencia investigadora existente, porque los artículos muestran fundamentalmente resultados en una única dirección. Otra práctica habitual es publicar un solo estudio de resultado positivo de forma "troceada" en distintos artículos, dividiéndolo por centros o por fases diferentes del estudio, alterando el orden de los autores para dar la impresión de que la evidencia defendida viene de muchos estudios y no de uno solo. Un ejemplo de ello, que recogen Mata y Ortiz (11), es un estudio sobre olanzapina del que aparecieron publicados más de 80 artículos.

Las guías terapéuticas de consenso, tan aparentemente recomendables, también han sido puestas en tela de juicio. Un estudio publicado en JAMA (12) analiza las relaciones entre los autores de distintas guías clínicas y la industria farmacéutica, encontrando que la mayor parte de ellos recibían dinero de la industria o trabajaban como asesores para ella. Otro trabajo más reciente publicado en Psychoterapy and Psychosomatics en 2009 (13) concluye señalando que el 90\% de los autores de tres de las principales guías de práctica clínica en psiquiatría, tenían vínculos financieros con las compañías que producían los fármacos que eran explícita o implícitamente recomendados en dichas guías como tratamientos para las respectivas enfermedades mentales. Ninguna de estas relaciones comerciales era revelada en las guías clínicas.

Es evidente que la formación médica continuada requiere una gran cantidad de fondos. Las distintas sociedades científicas e instituciones docentes solicitan el apoyo de las empresas farmacéuticas para llevar a cabo sus actividades, debido posiblemente al escaso apoyo recibido desde las administraciones públicas (14). Ya Mata y Ortiz señalaron el sesgo de contenidos que esta situación conlleva: la mayor parte de la inversión de la industria se dirige a actividades de corte biologicista y especialmente farmacológico, lo que provoca una progresiva asunción del paradigma biológico en psiquiatría (neuroquímico, en realidad) como el único existente en la inmensa mayoría de congresos, charlas, conferencias o publicaciones. Y además de este sesgo general de contenidos, muchas de estas actividades supuestamente 
ORIGINALES Y REVISIONES

de formación continuada son en realidad "publirreportajes" de nuevos fármacos o nuevas indicaciones (y, cada vez más, de nuevas enfermedades), donde la información que se facilita está tremendamente sesgada para favorecer los intereses de la compañía patrocinadora (15).

Son también importantes las repercusiones sobre la investigación que este entramado comercial lleva aparejadas. Hace unos quince años triunfaba la hipótesis serotoninérgica de la depresión, a partir de la llegada de la fluoxetina y su corte, por fin fármacos, se nos dijo, específicos, selectivos y bien tolerados (aunque paradójicamente dicha llegada coincidió con un aumento en la incidencia y prevalencia de la depresión, en vez de con una disminución de la misma). Esta hipótesis serotoninérgica decayó coincidiendo con la pérdida de las patentes de los ISRS y la salida al mercado de los antidepresivos duales como venlafaxina o duloxetina, que son vendidos como más eficaces ya que actúan en varios sistemas de neurotransmisión. La hipótesis monoaminérgica centrada en la serotonina nació y murió con el advenimiento y la caducidad de la patente del famosísimo y ya inserto en la cultura popular Prozac.

Nuestras reuniones científicas, publicaciones, guías clínicas y tratados llevaron a cabo de forma paulatina el cambio de denominación de los fármacos usados en psiquiatría: desaparecieron las categorías tradicionales que los describían a nivel funcional como tranquilizantes mayores, neurolépticos o estimulantes, para pasar a usar nombres como antipsicóticos o antidepresivos, que sugieren fármacos específicos para enfermedades específicas. Y luego, en otra genial estrategia comercial que ya se apunta, nos hablarán cada vez más de dimensiones y menos de categorías. Por lo tanto, como todo paciente (¿toda persona?) puntuará un determinado nivel en depresión, ansiedad o psicosis, se le podrán prescribir psicofármacos de todos los grupos a la vez. No como ya hacemos ahora, sino con una base (supuestamente) científica, como debe ser.

Citando de forma textual el excelente artículo de Mata y Ortiz: "Es de suponer que uno no estudia la carrera de medicina, aprueba el MIR y hace la residencia para acabar contando criterios diagnósticos y hacer una receta a continuación, pero una buena parte de los psiquiatras no hace mucho más. [...] esto es parte del presente de los psiquiatras y será el futuro de seguir las cosas así. Es muy difícil escapar del pensamiento único omnipresente en cursos, seminarios, revistas, investigaciones y viajes de placer, especialmente para las nuevas generaciones que se incorporan a la profesión con estas estrategias de mercado ya consolidadas".

Siguiendo con nuestro análisis de cómo la industria farmacéutica y su influencia (lo que el Dr. Sobrino Daza, Jefe de Servicio de Cardiología del Hospital Universitario La Paz, llama en un artículo de El País de 3 de mayo de 2010, siguiendo a Arnold Relman, "el complejo médico-industrial"), marca los contenidos y desarrollos científicos y tecnológicos en nuestra disciplina, apuntaremos unos co- 
mentarios acerca de la llamada Medicina Basada en la Evidencia (en más correcta traducción: medicina basada en indicios o pruebas). Ya nuestro grupo abordó este tema en un trabajo previo citado más arriba, que venía a sumarse a una corriente de opinión un tanto crítica sobre el uso y sobre todo el abuso de la MBE, señalando también las discrepancias entre tal posición teórica y la práctica psiquiátrica habitual. Un artículo de Spielman, de título revelador, publicado en el Bioethical Inquiry de 2010 (16) recoge numerosos documentos internos de la industria farmacéutica que sugieren, como comentamos antes, que los datos publicados disponibles pueden no ser representativos del total de datos acerca de sus productos. La industria y sus empresas de comunicación médica asociadas consiguen que lo publicado en la literatura médica sirva primariamente a sus intereses. La supresión o interpretación favorable de datos negativos (mediante supresión selectiva de datos o cambios a posteriori en las definiciones o criterios de análisis), así como el llamado "ghostwriting" al que también antes hicimos alusión, se han configurado en herramientas que utilizan a las revistas científicas para promocionar las ventas de sus productos, mientras que técnicas como el "disease mongering" (expansión de los límites reconocidos de una enfermedad para incluir expresiones subclínicas, limítrofes o incluso un rango normal de síntomas con el fin de incrementar la prescripción y venta de un determinado fármaco o terapia), al que luego nos referiremos, se usa también de forma sumamente eficaz para maximizar los beneficios económicos. Los autores concluyen que, aunque la medicina basada en pruebas es un noble ideal, la medicina basada en el marketing es la realidad habitual.

No queremos abandonar la cuestión de la MBE sin detenernos en unas reflexiones leídas recientemente: "No es sólo que la MBE afecte negativamente la calidad de la relación clínico-paciente sino que la reduce a una táctica neocapitalista para hacer negocios. [...] Su problema [de la MBE] deriva de una perversión epistemológica [...] profunda, resultante de la cosificación del hecho de prescribir y cuidar de las personas que sufren un trastorno mental. Esta identificación está estrechamente relacionada con las demandas de una economía neo-capitalista que precisa abrir nuevos mercados y crear nuevas necesidades consumistas. [...] Y en medio de esta locura, donde todo el mundo quiere hacer negocio, la vieja relación médico-paciente, y el paciente que sufre, han desaparecido para siempre". Poco se podría añadir a tan contundentes palabras. Su autor es el Profesor Germán E. Berrios, Catedrático de Psiquiatría de la Universidad de Cambridge (17).

Un editorial reciente del American Journal of Psychiatry (18) señala los conflictos de intereses que la relación entre la industria y los psiquiatras lleva aparejados, concluyendo que cada psiquiatra debe conocer, en las elecciones que hace, su propia responsabilidad en limitar dichos conflictos de intereses con el fin de preservar la integridad de nuestra disciplina. En relación con ello, queremos comentar un curioso artículo de Chimonas (19). Este estudio describe unos grupos de 
ORIGINALES Y REVISIONES

trabajo llevados a cabo con cinco o seis médicos y un moderador, centrados en la cuestión del conflicto de intereses planteado en la interacción con representantes de la industria farmacéutica (en visita médica, regalos, invitaciones y formación). Los médicos manifestaban reconocer la existencia de dicho conflicto de intereses, así como el hecho de que no por ello inhibían su interacción con los representantes comerciales. Mantener estas actitudes mutuamente contradictorias coloca a los médicos en una situación tipo de disonancia cognitiva. Tras este debate, los médicos actuaban utilizando una variedad de negaciones y racionalizaciones para el manejo de esta incómoda disonancia cognitiva: evitaban pensar acerca del conflicto de intereses, se mostraban en desacuerdo con que las relaciones con la industria afectaran su conducta médica, negaban tener responsabilidad en el problema, enumeraban técnicas para mantenerse imparcial y razonaban que los encuentros con los representantes eran educativos y beneficiaban a los pacientes. Los autores concluyen que, dada la facilidad para el manejo de la disonancia cognitiva, las medidas de autocontrol y manejo propio del inevitable conflicto de intereses, tal y como proponen la mayoría de las sociedades médicas, son inadecuadas. Afirman que es posible que sólo la prohibición de la interacción médico-representante sea efectiva, porque cualquier otra actuación es fácilmente neutralizada.

Un editorial de la revista de la Asociación Médica Canadiense (20) trata sobre la formación continuada de los médicos en dicho país. Tras destacar el desmesurado papel que juega la industria farmacéutica en dicha formación y referir los dilemas éticos y de otro tipo que este sistema plantea, se propone la adopción urgente de una serie de medidas para salvaguardar la independencia y el control de estas actividades por los propios profesionales, a los que también dedica cierta crítica: "Parte de la responsabilidad reside claramente en los médicos. Con los años, los poderosos alicientes farmacéuticos les han dado pie a creer que una fuerte participación de la industria no sólo es normal, sino también que tienen derecho a recibir beneficios. Esta cultura del derecho puede ser uno de los obstáculos más dificiles de superar".

Andrew Scull, profesor del Departamento de Sociología de la Universidad de California y conocido por sus múltiples publicaciones acerca de la psiquiatría, así como por sus críticas a La Historia de la Locura en la Época Clásica de Foucault, ha publicado un recomendable y polémico artículo en The Lancet (21). Habla acerca de la historia de la psiquiatría y su evolución, y concluye: "En los EEUU el Instituto Nacional de Salud Mental proclamó el decenio de 1990 "la década del cerebro”. Un reduccionismo simplista biologicista que cada vez más gobernó el gallinero psiquiátrico. Los pacientes y sus familias aprendieron a atribuir la enfermedad mental a la bioquímica defectuosa del cerebro, a defectos de la dopamina o a una escasez de serotonina. Este "bioparloteo" era tan profundamente engañoso y poco científico como el "psicoparloteo" al que reemplazó, pero como forma de 
comercialización no tuvo precio. Mientras tanto, la profesión psiquiátrica fue seducida y comprada con barcos cargados de financiación para la investigación. Donde una vez el psiquiatra había sido el más marginal de los médicos, existiendo en una zona de penumbra en los márgenes de la respetabilidad profesional, ahora eran los favoritos de los decanos de las facultades de medicina, con los millones y millones de las subvenciones y la recuperación de costos indirectos para ayudar a financiar la expansión del complejo médico-industrial. Y así el escándalo. El que paga al gaitero elige la canción y, en un grado extraordinario, el dinero de los fármacos ha llegado a dominar la Psiquiatría". En relación a esto y para completar este primer apartado dedicado a cómo la industria marca la hoja de ruta de nuestra investigación y docencia, del campo científico y tecnológico de nuestra disciplina, queremos citar también una palabras de Loren R. Mosher, coeditor de un libro imprescindible titulado Modelos de Locura (22), en la carta de renuncia que dirigió a la Asociación Psiquiátrica Americana (APA) en 1998: "En este punto de la historia, en mi opinión, la psiquiatría ha sido completamente comprada por las empresas farmacéuticas. La APA no podría continuar sin las compañias farmacéuticas apoyando sus reuniones, simposios, talleres, publicidad en revistas, grandes rondas de almuerzos, becas educativas sin restricciones, etc., etc. Los psiquiatras se han convertido en los siervos de las promociones de las compañías farmacéuticas. La APA, por supuesto, mantiene que su independencia y su autonomía no se ven comprometidas en esta enmarañada situación. [...] Pareciera que hubiésemos olvidado un principio básico: la necesidad de estar orientados a la satisfacción del paciente/cliente/consumidor. Yo siempre recuerdo la sabiduría de Manfred Bleuler: "Loren, nunca debes olvidar que eres el empleado de tu paciente". Al final, ellos determinarán si la Psiquiatría sobrevive o no en el mercado de los servicios".

Industria farmacéutica y nosografía.

Nuestro segundo apartado trata de lo que llaman González Pardo y Pérez Álvarez (23) La invención de trastornos mentales, título de un libro más que recomendable. Relatan cómo se han inventado determinados trastornos o cómo se han sobredimensionado otros. Siguiendo algunos de sus ejemplos, recordamos cómo el trastorno por estrés postraumático fue creado como categoría diagnóstica para el DSM-III en 1980, ante la presión política y social por dar un nombre a los síntomas de ansiedad y depresión que presentaban los veteranos de Vietnam. Una vez categorizado, el trastorno se independiza de su origen y ha alcanzado en la actualidad rango de epidemia para multitud de sufrimientos secundarios a traumas vividos $\mathrm{u}$ olvidados, mezclando de forma perversa el concepto de víctima con el de enfermo. 
ORIGINALES Y REVISIONES

Naturalmente, al no prestar atención a la creación social de esta categoría, las nuevas generaciones de profesionales (y no tan nuevas) la tratan como si fuera un ente natural como la tuberculosis o el sarampión.

Otro ejemplo que comentan González y Pérez es el de la depresión, una entidad clínica en constante aumento. Gracias a múltiples campañas para localizar "depresiones ocultas" a las que poder aplicar "tratamientos eficaces", vamos construyendo una sociedad en la que estar triste por cualquier motivo, consciente o no, se ha convertido en sinónimo de estar enfermo y requerir tratamiento (usualmente psicofarmacológico). Los profesionales nos quejamos de la saturación de nuestras consultas pagadas con dinero público, pero ante cualquier duelo, tristeza o adaptación difícil, diagnosticamos algún subtipo de depresión de los que pone a nuestro alcance el sistema clasificatorio actual. Hasta 1980, la depresión era un diagnóstico poco frecuente, calculándose que del orden de 50 a 100 personas por millón la sufrían, mientras que estimaciones de esta década que termina la sitúan en cien mil por millón (24). Este "boom" de la depresión coincidió, curiosamente, con la aparición del DSM-III y los nuevos antidepresivos, mucho más caros que los antiguos. En palabras de González y Pérez, "Lo que ha ocurrido en la sociedad actual es tanto una sensibilización como una intolerancia para todo lo que suponga bajo humor y tristeza, como si la condición natural fuera la "euforia perpetua". De hecho, el término depresión capitaliza clínicamente lo que de otra manera serían experiencias normales de la vida con un curso natural".

Otra gran creación ha sido la fobia social. Es un buen ejemplo de cómo problemas sociales o personales se han convertido en problemas médicos (25). La promoción de la fobia social como trastorno ha seguido un cuidadoso sistema de marketing que, buscando promover un medicamento, ha promovido primero un trastorno. La compañía farmacéutica Glaxo-SmithKline contrató a la agencia de publicidad Cohn \& Wolfe para promover el trastorno de ansiedad social como una enfermedad grave, que sería tratada con su molécula paroxetina (Paxil $®$ - Seroxat $($ ) $)$. El primer paso consiste en sensibilizar a la población general ante este trastorno, con anuncios y noticias que hablan de lo frecuente que es ("según los expertos") y los estragos que causa. Se dice que, hasta ahora, ha sido una enfermedad infradiagnosticada, que se sufría sin ayuda, pero que ahora tiene tratamiento. Simultáneamente, se educa al médico a través de diversos medios (visitadores, propaganda en revistas científicas, congresos y jornadas sobre el tema...) anunciando la nueva enfermedad y su remedio. Se establece la necesidad en la gente para el nuevo fármaco y se crea el deseo de recetarlo en los clínicos. Una vez sensibilizada la población, se recomienda "consulte a su médico" y donde teníamos una solución en busca de un problema, se ha conseguido que sea el problema creado el que busque la solución. La prevalencia de la fobia social a principios de los años 80 era del 2,75\% y pasó, en diez años, a un 13,3\% (26). 
Siguiendo con ejemplos de trastornos inventados o, más bien, inflacionados (en el sentido de bajar el umbral diagnóstico para aumentar la cantidad de fármaco prescrita, al coste de tratar como enferma gente previamente considerada sana), podríamos señalar, ya desde nuestra propia experiencia clínica, la epidemia de trastorno bipolar que vivimos, en la cual cualquier período en el que una persona se presente exaltada, inquieta o más alegre de lo habitual, o lleve a cabo conductas de gasto o promiscuidad que su familia considere no adecuadas, es diagnosticada de episodio maníaco (siendo muy fácil, con los niveles de corte actuales, encontrar episodios depresivos previos prácticamente en la biografía de cualquiera). El diagnóstico de trastorno bipolar está hecho, con lo que lleva aparejado de cronicidad y de medicación eutimizante y muchas veces antipsicótica y ansiolítica de por vida. Y, por supuesto, no estamos dudando de la existencia del trastorno bipolar o psicosis maniacodepresiva, pero sí señalando cómo, en nuestra opinión, se sobrediagnostica, con los perjuicios que eso puede conllevar a nuestros pacientes. Y señalar también una curiosidad: cómo hemos pasado en una o dos décadas a dejar de hablar de bouffée delirante o psicosis aguda y referirnos a cuadros clínicos de ese tipo como "primeros brotes" o "primeros episodios" de esquizofrenia. No hace falta señalar la obviedad de que el término "primero" inaugura una lista y hace inevitable que el clínico, el paciente y su familia den por hecho que habrá un segundo. Esta visión incide en el estigma y la cronificación y crea unas expectativas negativas, además de provocar la medicación de por vida a pacientes que tal vez no la necesitaran. Y nuevamente aclaramos: no estamos en contra de la medicación psiquiátrica y creemos necesario el tratamiento de mantenimiento neuroléptico en esquizofrenia, a dosis adecuadas y buscando el beneficio del paciente en términos de control de la angustia y de los fenómenos que la provocan. Pero nos horroriza la posibilidad de estar medicando de por vida con fármacos no inocuos a gente que no se beneficie de ello.

Allen Frances fue Jefe de Grupo de Tareas del DSM-IV (poco sospechoso, en principio, de intereses antipsiquiátricos o tendencias revolucionarias) y ha publicado recientemente un texto muy crítico con el borrador de nuestro próximo DSM-V (27). Habla este autor acerca de los riesgos que entrañará el DSM-V en comparación con el IV, señalando la existencia de nuevos diagnósticos que podrían ser extremadamente comunes entre la población general (especialmente después del marketing de una siempre alerta industria farmacéutica), así como umbrales diagnósticos más bajos para muchos trastornos ya existentes. Siguiendo su artículo, el DSM-V (aún un borrador) podría crear decenas de millones de falsos positivos, exacerbando los problemas creados por un ya demasiado inclusivo DSM-IV. Excesivos tratamientos masivos con medicaciones innecesarias, caras y a menudo dañinas. Los nuevos diagnósticos problemáticos que comenta son: el síndrome de riesgo de psicosis (según Frances, 70-75\% de falsos positivos), el trastorno mixto 
ORIGINALES Y REVISIONES

de ansiedad depresiva (los autores de este trabajo lo hemos tenido, suponemos que la mayoría de los lectores también), el trastorno cognitivo menor (¿la vejez?), el trastorno por atracones (bastará una vez a la semana durante tres meses; uno de los autores lo tiene), el trastorno disfuncional del carácter con disforia (¿la mala leche?; los dos autores la tienen), el trastorno coercitivo parafílico (con lo cual, y esto ya no hace gracia, muchos violadores ya no serían criminales sino enfermos), el trastorno de hipersexualidad (sin comentarios), las adicciones conductuales (que podrán incluir en la categoría de adicciones a sustancias, por ejemplo, el juego patológico o bien adicciones no especificadas, como a comprar, a internet, a los videojuegos... y no debemos olvidar que cuando todo es enfermedad, ya no queda nada de responsabilidad). En lo referente a umbrales más bajos para categorías ya existentes, Frances señala que ello ocurre para el trastorno de déficit de atención con o sin hiperactividad, el trastorno de adicción, el trastorno del espectro del autismo, la medicalización del duelo normal, la pedohebefilia (¿el sexo con individuos de 14 ó 15 años debe ser una cuestión legal o psiquiátrica?, ¿tener relaciones con un adolescente menor de edad debe entenderse como una conducta regulable por la ley en cada sociedad o como un síntoma de enfermedad mental?).

Para cerrar este apartado, querríamos citar unas palabras de Carlos Castilla del Pino (28): "La psiquiatrización de la sociedad está, en gran parte, promovida por las empresas farmacéuticas. Eso debe saberse. [...] hay una inflación de la enfermedad. Me dedico a la Psiquiatría desde hace sesenta y seis años y los llamados bipolares son lo que antes conocíamos por ciclotímicos, personas que pasan de la euforia a la depresión. Nunca se habían diagnosticado tantos casos como ahora, cualquiera es calificado de bipolar. Además, se ha introducido otro concepto peligrosísimo, que es el de la llamada "comorbilidad", es decir, la coexistencia de dos enfermedades, aunque en la práctica es la coexistencia de dos síntomas [...] Las depresiones con tristeza inmotivada e inhibición son tan escasas como en la época en que no había bipolares y se diagnosticaba ciclotimia. Es otro abuso".

Industria farmacéutica, eficacia terapéutica y seguridad.

Nos centraremos ahora en los productos que vende la industria farmacéutica, como un arsenal a nuestra disposición "seguro y eficaz". La principal información que le llega al psiquiatra prescriptor proviene directamente de la misma industria, a través de estudios presentados por los visitadores médicos, de charlas o jornadas organizadas o patrocinadas por los laboratorios o por estudios en revistas científicas diseñados y realizados por encargo o bajo patrocinio de la industria. Evidentemente y como señalamos antes, el sesgo es inevitable desde diversos lados. 
Pero es cierto que cada vez más encontramos trabajos independientes por parte de administraciones públicas o investigadores autónomos que estudian, desde la independencia respecto de los intereses del marketing empresarial, los psicofármacos que recetamos. Pondremos algunos ejemplos.

PLoS Medicine (29) publica un metaanálisis con 5.133 pacientes en 2008 que concluye que, excepto en depresiones graves, los antidepresivos fluoxetina, venlafaxina, paroxetina y nefazodona no son más eficaces que placebo. En 2003, JAMA (30) publica un ensayo clínico aleatorizado y doble ciego con 309 pacientes a doce meses que concluye que no hay diferencias en eficacia o tolerancia entre olanzapina y haloperidol, ambos con medicación anticolinérgica acompañante. Los autores afirman que olanzapina no presentaba ventajas respecto a haloperidol en cumplimiento, síntomas, extrapiramidalismo o calidad de vida y que sus beneficios en menor acatisia y mejora de la cognición debían equilibrarse con el aumento de peso y el mayor coste. El estudio CATIE $(31,32)$ fue un ensayo clínico aleatorizado y doble ciego que incluyó 1.493 pacientes, el cual no demostró ventajas de cuatro antipsicóticos atípicos (olanzapina, risperidona, quetiapina, ziprasidona) frente a perfenazina en lo referente a medida de síntomas, efectos neurológicos secundarios, calidad de vida, empleo o función neuropsicológica. El estudio CUtLASS (33) fue un ensayo clínico multicéntrico aleatorizado de 227 pacientes a un año, el cual descartó la hipótesis previa de que los pacientes tratados con antipsicóticos atípicos tendrían una mejora en su calidad de vida a lo largo del año de estudio. El grupo con antipsicóticos típicos mostró una tendencia, no estadísticamente significativa, hacia mayor mejoría en las escalas de calidad de vida y síntomas. Tampoco se observaron ventajas de los antipsicóticos atípicos en cuanto a efectos secundarios.

Un estudio observacional publicado en JAMA (34) sobre unos 300 niños y adolescentes tratados con un nuevo antipsicótico durante doce semanas encontró que el $84 \%$ de los tratados con olanzapina aumentaron de peso como mínimo un 7\% del valor inicial y casi un 30\% presentaron dislipemia. El British Medical Journal (35) publicó recientemente un estudio observacional el cual concluye que la paroxetina reduce la metabolización del tamoxifeno y daría lugar a un aumento de la mortalidad por cáncer de mama. También en el $B M J(36)$ encontramos un trabajo que observa cómo el uso de sertralina o citalopram durante el primer trimestre de la gestación se asocia a un aumento del riesgo de defectos septales cardíacos en la descendencia.

Un estudio de casos y controles publicado en el American Journal of Psychiatry (37) afirma que los fármacos psicoestimulantes que se utilizan en el tratamiento del trastorno por déficit de atención-hiperactividad se asocian a un aumento del riesgo de muerte súbita en pacientes jóvenes. El aumento del riesgo se estimó en unas siete veces para los usuarios de metilfenidato. Otro estudio (38) sobre el 
ORIGINALES Y REVISIONES

uso de estos fármacos encuentra asociación con alucinaciones y otros síntomas psicóticos. Un tercer trabajo (39) encuentra que el tratamiento con estimulantes en la infancia provoca retrasos estadísticamente significativos de peso y altura. En este estudio no se encontró evidencia estadísticamente significativa de la atenuación de estos déficits con el tiempo.

Un artículo de cohortes retrospectivo publicado en el New England Journal of Medicine (40) afirma que el riesgo de arritmias ventriculares graves y muerte súbita cardíaca en el tratamiento con antipsicóticos atípicos es al menos tan alto como con los tradicionales. Estamos hablando de 2,9 muertes por 1000 pacientes/ año, lo que significa una incidencia baja pero no rara y, en ausencia de una clara efectividad del fármaco, la posibilidad que existe de dañar es inaceptable.

La Agencia Española del Medicamento y Productos Sanitarios (AEMPS), recoge en sus notas $(41,42)$ datos que identifican un riesgo incrementado de malformaciones congénitas cardiovasculares asociado con el uso de fluoxetina en el primer trimestre del embarazo; asociación entre hipertensión pulmonar en neonatos y el uso de citalopram, duloxetina, escitalopram, fluoxetina, fluvoxamina, mirtazapina, paroxetina, sertralina y venlafaxina durante el embarazo, recomendándose precaución en su uso, especialmente en la fase final del mismo; y por último, estudios epidemiológicos comentados en estas notas muestran un aumento del riesgo de fracturas óseas con el uso de antidepresivos tricíclicos o ISRS, por mecanismo desconocido.

La Ficha de Evaluación Terapéutica del Servicio Navarro de Salud para la agomelatina (43) dice textualmente: "peor que los antidepresivos de elección, hasta 15 veces más caro y de dudosa seguridad hepática".

Estos ejemplos muestran cómo es necesario acudir a fuentes distintas a las patrocinadas u organizadas por la industria para tener una información más completa de los fármacos que prescribimos a nuestros pacientes, tanto en lo que se refiere a su eficacia como a su seguridad. No obstante, existen estudios independientes fácilmente accesibles gracias, por ejemplo, a diversas páginas web de administraciones públicas. Tenemos, por mencionar algunos, el "Butlletí Groc", editado por la Fundació Institut Catalá de Farmacología (http://w3.icf.uab.es/notibg/index.php); las "Fichas de Evaluación Terapéutica", que proporciona el Servicio Navarro de Salud (http://www.navarra.es/ home_es/Temas/Portal+de+la+Salud/Profesionales/ Documentacion $+y+$ publicaciones/Publicaciones + tematicas/Medicamento/FET/) o las publicaciones "Bolcan" e "Infarma" de la Dirección General de Farmacia del Servicio Canario de la Salud (http://www2.gobiernodecanarias.org/sanidad/scs/ organica.jsp ?idCarpeta $=d 05 b 6 d b b-31 a d-11 d f-b 5 d 7-a 3 a 2 f b c b 2 f 35)$. 


\section{Industria farmacéutica y gasto sanitario.}

Nuestro cuarto apartado sobre la influencia de la industria farmacéutica en la psiquiatría se dedica a la cuestión del gasto sanitario. Vivimos una época marcada por una crisis económica global, que ha disparado las cifras de paro y puesto en serios aprietos a los estados, hasta el punto de bajar sueldos públicos, congelar pensiones, parar infraestructuras y comenzar a hablar de financiación sanitaria en términos de copago o similares, con un evidente declive, si no algo peor, del estado de bienestar tal como lo conocemos. En este contexto, las cifras de gasto sanitario achacables a los fármacos, en nuestro caso a los psicofármacos, crecen de año en año. A los médicos nos gusta pensar y decir que recetamos lo mejor a nuestros pacientes sin importar lo que cueste. Es una posición loable con la que nosotros estamos básicamente de acuerdo. El problema surge cuando lo que se receta no es mejor sino sólo más caro, más caro por ser más nuevo, lo que a su vez conlleva que sea más desconocido en lo referente a su seguridad. Y el dinero que manejamos en nuestras prescripciones es público, limitado y, lamentablemente, será cada vez más escaso. No trabajamos en un sistema que se pueda hacer cargo de un gasto infinito, y lo usado en un sitio ya no podrá emplearse en otro. Tenemos que ser gestores del gasto que provocamos, ya que hay que pensar en el paciente que tenemos delante, pero también en los que vendrán dentro de cinco, diez o veinte años, que necesitarán que no hayamos arruinado el sistema público de salud que pagamos y sostenemos entre todos. Se nos puede acusar de catastrofistas y exagerados. Ojalá lo seamos.

La industria farmacéutica justifica los elevados precios de los nuevos fármacos en su potente inversión en investigación de nuevas moléculas. Sin embargo, en el año 2000, las principales empresas farmacéuticas destinaron un $12 \%$ de su presupuesto a investigación y desarrollo, frente a un $30 \%$ destinado al marketing $(44,45)$. La aparición de los fármacos genéricos con el mismo principio activo pero un precio menor es una dura competencia cuando caduca la licencia de la patente de un producto. Por ello, los laboratorios van comercializando sucesivamente nuevas presentaciones (comprimidos, solución, bucodispersable...) para que, cuando caduque la patente de la primera presentación, mantener la de las otras (simultáneamente, los visitadores y los expertos que nos trae la industria en jornadas y congresos nos informan de que la nueva presentación es fabulosa y la anterior es pésima, y el fármaco en solución que mandábamos habitualmente es desechado por el bucodispersable, por ejemplo, sin que tengamos en cuenta la diferencia de precio $\mathrm{y}$, por consiguiente, de gasto de dinero público que ello supone). Otra estrategia habitual es, cuando va a expirar la patente, se modifica ligeramente la molécula y tenemos un nuevo fármaco. Y a pesar de que no haya estudios de comparación con el original, asumimos, porque así se nos dice, que como es nuevo será mejor. Y no 
ORIGINALES Y REVISIONES

nos preocupamos de que el precio sea mucho más elevado ni de que realmente sea un fármaco menos estudiado y, por tanto y en buena lógica, su seguridad sea más indeterminada. Ejemplos en psiquiatría de estas prácticas son abundantes.

Hace poco asistimos al lanzamiento de la quetiapina de liberación prolongada, en coincidencia con la caducidad de la patente de la quetiapina de liberación inmediata comercializada previamente. En un "Informe de Evaluación del Medicamento" publicado por el Govern de les Illes Balears (46) se analizan los estudios existentes y se concluye que la nueva presentación no ha demostrado su superioridad sobre la antigua y que el supuesto beneficio de mayor adherencia por poder tomar sólo una dosis diaria no se ha determinado en ningún ensayo clínico. La conclusión textual del análisis es "no supone ningún avance terapéutico". Aunque el gasto sí que avanza al hacer el cambio de una a otra. En "Notas Farmacoterapéuticas", publicación del Servicio Madrileño de la Salud (47), se analizan, entre otros estereoisómeros, escitalopram frente a citalopram. Las conclusiones parecen claras: "aunque [...] parece aportar a priori aspectos beneficiosos, como un inicio de acción más rápido, los estudios comparativos con citalopram muestran unas diferencias de relevancia clínica discutible. [...] su aparición en el mercado coincide con la pérdida de la patente de la molécula original, [...] lo que parece corresponder a una estrategia comercial de mantenimiento de cuota del mismo". Recientemente, tras caducar la patente de risperidona, se inicia la comercialización de paliperidona. El Servicio Navarro de Salud publicó una "Ficha de Evaluación Terapéutica" (48) que afirma: "no supone avance terapéutico", recomendando que "en los casos en que esté indicado el uso de risperidona o paliperidona, el fármaco de elección es la risperidona".

La Dirección General de Farmacia del Servicio Canario de la Salud difundió recientemente una nota (49) recomendando la prescripción por principio activo y señalando las siete moléculas que representan mayor gasto para el sistema, de las que cuatro son de uso psiquiátrico: risperidona, olanzapina, venlafaxina y escitalopram. Calcula que el ahorro en ellas, si estas cuatro moléculas se recetaran por principio activo, oscilaría entre el $36 \%$ y el $69 \%$.

Y llegamos a otra de las estrategias de la industria para aumentar sus beneficios: la duda que siembran acerca de la bioequivalencia entre genéricos y marcas y el famoso $20 \%$ de variación, intentando engañar al médico para que piense que ese $20 \%$ es variación en cantidad de principio activo, cosa que es falsa. Se trata de un parámetro que sirve para comparar, no sólo genérico y marca, sino también distintas presentaciones de la misma marca (cuando dejamos de mandar la solución y empezamos con el bucodispersable, es porque se ha aprobado la bioequivalencia entre ambas presentaciones en base a los mismos estudios de bioequivalencia que se usan para los genéricos). Diversas notas realizadas por administraciones públicas, como por ejemplo el Servicio Canario de la Salud (50) o el Servicio de Farma- 
cia del Hospital 12 de Octubre (51), explican claramente cómo no hay diferencias en cantidad de principio activo y, por lo tanto, en eficacia o seguridad entre dos productos bioequivalentes, sean genérico y marca, o dos presentaciones de una marca. Nos parece que el tema está claro para quien quiera leerlo. Un estudio publicado en 2004 (52) con 5.000 genéricos no encontró ningún caso de fallo terapéutico o toxicidad que pudiera atribuirse a la bioequivalencia respecto de los fármacos de referencia. Se suele argumentar que la prescripción de genéricos podría provocar la caída de grandes laboratorios que son los que llevan el peso de la investigación farmacológica. Es relativo, porque la investigación actual realmente no está encaminada a nuevos descubrimientos, sino más bien a unos cambios químicos cosméticos en moléculas ya conocidas (véase el ejemplo de la paliperidona, el escitalopram o todos los derivados del omeprazol), más baratos de realizar y mucho más rentables a la hora de vender. Cerrar esa puerta no cayendo en la trampa de las moléculas me too (que se lanzan con el mensaje de ser al menos tan eficaces como, basándose en estudios de tamaños muestrales de baja potencia estadística que, lógicamente, no hallan diferencias significativas con el fármaco control), y prescribiendo por genéricos, posiblemente llevara a la industria a la necesidad de investigar líneas nuevas que puedan aportar algo distinto en terapéutica, algo que en psiquiatría hace ya más de una década, por ser conservadores, que no se produce. La misma dinámica de este mercado hace que no se investigue en nuevos fármacos para el paludismo $\mathrm{u}$ otras enfermedades tropicales que asolan los países pobres, precisamente porque son pobres y ahí no hay beneficio esperable. Los países africanos no pueden pagar los antirretrovirales para el sida, ni se les deja comercializarlos saltándose la patente, en aras del sacrosanto beneficio empresarial, que luego se usa mucho más en promocionar productos ante los médicos que en investigar nada.

Y para completar este apartado referente al gasto farmacéutico, sólo un último ejemplo que no tiene que ver directamente con la psiquiatría, sino con la famosa gripe A. Leemos en una noticia de El País (53) que un informe clave de la OMS ocultó los vínculos financieros entre sus expertos y las farmacéuticas Roche y Glaxo (fabricantes de tamiflu ${ }^{\circledR}$ y relenza ${ }^{\circledR}$, los antivirales contra el H1N1). Este informe instó a los gobiernos a acumular reservas de esos medicamentos por valor de 4.900 millones de euros. Y ahora, a congelar pensiones y bajar sueldos si hay suerte y si no, a la cola del paro.

\section{Conclusiones.}

¿Y qué concluir de todo esto? No se trata de demonizar a la industria ni a los médicos, sino de describir un estado de cosas. De tener conocimiento, que es la 
ORIGINALES Y REVISIONES

primera condición del poder. Y, como psiquiatras y profesionales de la salud mental, debemos recuperar el poder sobre nuestro trabajo. Creemos que otra Psiquiatría puede ser posible y, sin duda, que es necesaria. Una Psiquiatría que se dedique al estudio de su objeto (la mente, la conducta, la locura o como queramos llamarlo...) sin injerencias de intereses comerciales que sesguen nuestra información. Una Psiquiatría capaz de ponerse límites a sí misma y no pretender tratarlo todo, sabiendo que la normalidad, en la consulta psiquiátrica, muchas veces no mejora sino se cronifica, y que si tratas a alguien sano como un enfermo, lo más probable es que acabe viéndose, sintiéndose y comportándose como un enfermo. Una Psiquiatría que se centre, sobre todo, en el loco y el enfermo, en su sufrimiento, y deje a los cuerdos y sanos luchar por su felicidad sin falsos remedios. Una Psiquiatría que emplee sus tratamientos farmacológicos y psicoterapéuticos cuando estén indicados y de la forma adecuada, con información científica no sesgada. Una Psiquiatría que no colabore en arruinar un sistema público de salud que debe ser sostenible, porque de ello depende nuestro futuro y el de nuestros hijos. Y si tal Psiquiatría es posible, debemos intentar hacerla real.

En línea con plataformas como No Gracias (www.nogracias.eu), los autores hemos decidido cortar nuestros vínculos con la industria farmacéutica. Hemos hecho estudios para ellos y cobrado por ellos (ahora la última tendencia es que, además de pagarte por recoger unos datos, te ponen de autor corporativo, con lo que acabarán decidiendo también nuestro currículum y quién ocupa o no una plaza en la sanidad pública), hemos viajado a congresos y jornadas diversas, hemos ido a múltiples comidas y cenas "de trabajo"... Todo ello en el convencimiento de que tal conducta no afectaba a nuestra prescripción, que éramos capaces, pese a ello, de mantener la independencia. Pero descubrimos que no era así. Descubrimos que nos costaba prescribir un genérico, que nos era difícil no recetar la última molécula presentada... Y no pretendemos hacer apología de nuestra posición. No conocemos psiquiatras corruptos que indiquen tal fármaco a cambio de tal recompensa. Y creemos que puede haber compañeros que sean capaces de mantener su independencia a pesar de ese contacto con la industria, pero nosotros no pudimos mantenerla, y ahora queremos recuperarla. Cortamos lazos con la industria porque también, y es parte de la trampa, sus incentivos te acostumbran a un nivel de vida por encima del que el sueldo de nuestra profesión, en este país, debería permitirnos: viajes al extranjero todos los años, hoteles de cuatro y cinco estrellas, comidas y cenas en buenos restaurantes, libros gratis... Y cuesta renunciar a ello pero, en nuestro caso, lo hacemos. Hemos estado en la trinchera y, lógicamente, no hemos podido evitar mancharnos de barro, así que nos salimos.

Pero creemos que la solución no está sólo en manos de los profesionales. La solución pasa por las administraciones públicas, que deberían responsabilizarse de la investigación y la formación, hasta ahora abandonadas en manos de la industria, 
así como fijarse en qué fármacos el sistema público debe financiar y cuáles no, por no aportar nada nuevo. La solución pasa por las asociaciones profesionales, que deberían fijar precios para sus actos científicos que no obligaran a un patrocinio. La solución pasa por las direcciones de los centros públicos en que trabajamos, que deberían limitar y controlar (¿prohibir?) el acceso de visitadores médicos. Y, por supuesto, la solución pasa por cada psiquiatra y cada profesional, que debería, deberíamos, reflexionar sobre nuestras teorías y nuestras prácticas, parándonos a pensar qué tipo de Psiquiatría queremos.

\section{BIBLIOGRAFÍA:}

(1) Mata Ruiz I, Ortiz Lobo A, Industria farmacéutica y psiquiatría, Rev Asoc Esp Neuropsiq, 2003; 86: 49-71.

(2) García-Valdecasas Campelo J, Vispe Astola A, Tobías Imbernón C, Hernández González M, De la (curiosísima) relación entre la Medicina Basada en la Evidencia y la práctica psiquiátrica en nuestro entorno, Rev Asoc Esp Neuropsiq, 2009; 104: 405-421.

(3) Moynihan R, Heath I, Henry D, Selling sickness: the pharmaceutical industry and disease mongering, BMJ, 2002; 324: 886-891.

(4) Smith R, Maintaining the integrity of the scientific record (editorial), BMJ, 2001; 323: 588.

(5) Sponsorship, authorship, and accountability (editorial), The Lancet, 2001; 358: 854-856.

(6) Just how tainted has medicine become? (editorial), The Lancet, 2002; 359.

(7) Scandal of scientist who take money for papers ghostwritten by drug companies, The Guardian, 7 de febrero de 2002.

(8) The PLoS Medicine Editors, Ghostwriting: The Dirty Little Secret of Medical Publishing That Just Got Bigger, PLoS Med, 2009, 6 (9): e1000156. doi: 10.1371/journal.pmed. 1000156.

(9) Gilbody SM, Song F, Eastwood AJ, Sutton A, The causes, consequences and detection of publication bias in psychiatry, Acta Psychiatr Scand, 2000; 102: 241-249.

(10) Turner EH, Matthews AM, Linardatos E, Tell RA, Rosenthal R, Selective Publication of Antidepressant Trials and Its Influence on Apparent Efficacy, N Engl J Med, 2008, 358; 3: 252-260.

(11) Mata Ruiz I, Ortiz Lobo A, Sesgos y dificultades en la investigación de los nuevos antipsicóticos, Rev Asoc Esp Neuropsiq, 2001; 79: 57-74.

(12) Couhdry NK, Stelfox HT, Detsky AS, Relations between Authors of clinical practice guidelines and the pharmaceutical industry, JAMA, 2002; 287: 612-617.

(13) Cosgrove L, Bursztajn HJ, Krimsky S, Anaya M, Walker J, Conflicts of interest and disclosure in the American Psychiatric Association`s Clinical Practice Guidelines, Psychother Psychosom, 2009; 78: 228-232.

(14) Ferrán Mercadé M, La financiación de las sociedades científicas y la industria farmacéutica, Atención Primaria, 2002; 29: 327-328.

(15) Haayer F, Rational prescribing and sources of information, Soc Sci Med, 1982; 16: 20172023.

(16) Spielmans GI, Parry PI, "From Evidence-based Medicine to Marketing-based Medicine: 
ORIGINALES Y REVISIONES

Evidence from Internal Industry Documents, Bioethical Inquiry, 2010; 7: 13-29.

(17) Berrios GE, On evidence-based medicine, disponible en: http:// www.psicoevidencias.es/ Novedades/Editorial/on-evidence-based-medicine.html

(18) Freedman R, Lewis DA, Michels R, Pine DS, Schultz SK, Tamminga CA, et al, Conflict of Interest. An issue for every psychiatrist, Am J Psychiatry, 2009; 166: 274.

(19) Chimonas S, Brennan TA, Rothman DJ, Physicians and Drug Representatives: Exploring the Dynamics of the Relationship, J Gen Intern Med, 2007; 22(2): 184-190.

(20) Hébert PC, The need for an Institute of Continuing Health Education, CMAJ, 2008; 178 : 805-806.

(21) Scull A, A psychiatric revolution, The Lancet 2010; 375(9722): 1246-1247.

(22) Read J, Mosher R, Bentall R. Modelos de locura. Herder, Barcelona, 2006.

(23) González Pardo H, Pérez Álvarez M. La invención de trastornos mentales. Alianza Editorial, Madrid, 2007.

(24) Healy D. Let them eat Prozac. The unhealthy relationship between the pharmaceutical industry and depression. Nueva York: New York University Press, 2004.

(25) Moynihan R, Cassels A. Selling sickness. How the world`s biggest pharmaceutical companies are turning us into patients. Nation Books, Nueva York, 2005.

(26) Horwitz AV. Creating mental illness. University Chicago Press, Chicago, 2002.

(27) Frances A, Opening Pandora's Box: The 19 Worst Suggestions For DSM5, Psychiatric Times, 2010, Feb 11, disponible en: http:// www.psychiatrictimes.com/dsm/content/article/10168/1522341? pageNumber $=2$

(28) La industria farmacéutica promueve, en parte, la psiquiatrización social. Entrevista de J.L. Argüelles a Carlos Castilla del Pino, en La Nueva España, 19 de octubre de 2006.

(29) Kirsch I, Deacon BJ, Huedo-Medina TB, Scoboria A, Moore TJ, Johnson BT, Initial severity and antidepressant benefits: a meta-analysis of data submitted to the Food and Drug Administration, PLoS Med, 2008, 5, 2, e45 doi: 10.1371/journal.pmed. 0050045.

(30) Rosenheck R, Perlick D, Bingham S, Liu-Mares W, Collins J, Warren S, et al, for the Department of Veterans Affairs Cooperative Study Group on the Cost-Effectiveness of Olanzapine, Effectiveness and cost of olanzapine and haloperidol in the treatment of schizophrenia, JAMA, 2003, 290: 26932702.

(31) Lieberman JA, Stroup TS, McEvoy JP, Swartz MS, Rosenheck RA, Perkins DO, et al, for the CATIE Investigators, Effectiveness of antipsychotic drugs in patients with chronic schizophrenia: primary efficacy and safety outcomes of the Clinical Antipsychotic Trials of Intervention Effectiveness (CATIE) Schizophrenia Trial, N Engl J Med, 2005, 353: 1209-1223.

(32) Swartz MS, Perkins DO, Stroup TS, Davis SM, Capuano G, Rosenheck RA, et al, Effects of antipsychotic medications on psychosocial functioning in patients with chronic schizophrenia: findings from the NIMH CATIE Study, Am J Psychiatry, 2007, 164: 428-436.

(33) Jones PB, Barnes TR, Davies L, Dunn G, Lloyd H, Hayhurst KP, et al, Randomized controlled trial of effect on quality of life of second generation versus first generation antipsychotic drugs in schizophrenia - CUtLASS1, Arch Gen Psychiatry, 2006, 63: 1079-1087.

(34) Correll CU, Manu P, Olshanskiy V, Napolitano B, Kane JM, Malhotra AK, Cardiometabolic risk of second-generation antipsychotic medications during first-time use in children and adolescents, JAMA, 2009; 302: 1765-73. 
(35) Kelly CM, Juurlink DN, Gomes T, Duong-Hua M, Pritchard KI, Austin PC, et al, Selective serotonin reuptake inhibitors and breast cancer mortality in women receiving tamoxifen: a population based cohort study, $B M J, 2010 ; 340$ : c693.

(36) Pedersen LH, Henriksen TB, Vestergaard M, Olsen J, Bech BH, Selective serotonin reuptake inhibitors in pregnancy and congenital malformations: population based cohort study, BMJ, 2009; 339: b3569.

(37) Gould MS, Walsh BT, Munfakh JL, Kleinman M, Duan N, Olfson M, et al, Sudden death and use of stimulant medications in youths, Am J Psychiatry, 2009; 166: 992-1001.

(38) Mosholder AD, Gelperin K, Hammad TA, Phelan K, Johann-Liang R, Hallucinations and Other Psychotic Symptoms Associated With the Use of Attention-Deficit/Hyperactivity Disorder Drugs in Children, Pediatrics, 2009; 123 (2): 611-616 (doi:10.1542/peds.2008-0185).

(39) Faraone SV, Biederman J, Morley CP, Spencer TJ, Effects of stimulants on height and weight: a review of the literature, J Am Acad Child Adolesc Psychiatry, 2008; 47(9): 994-1009.

(40) Wayne AR, Chung CP, Murray KT, Hall K, Stein CM, Atypical Antipsychotic Drugs and the Risk of Sudden Cardiac Death, N Engl J Med, 2009; 360: 225-235.

(41) Agencia Española del Medicamento y Productos Sanitarios (AEMPS), Algunos datos de seguridad sobre antidepresivos y embarazo, disponible en: http://www.aemps.es/actividad/notaMensual/ docs/2010/inforMensual-feb10.pdf.

(42) Agencia Española del Medicamento y Productos Sanitarios (AEMPS), Datos de seguridad sobre antidepresivos e incremento de riesgo de fracturas óseas, disponible en: http://aemps.es/actividad/ notaMensual/docs/2010/infor Mensual -marzo10.pdf.

(43) Servicio Navarro de Salud, Agomelatina, Ficha de Evaluación Terapéutica $n^{\circ} 3$, 2010, disponible en: http://www.navarra.es/home_es/Temas/Portal+de+la+ Salud/Profesionales/Documentacion+y+publicaciones/Publicaciones+tematicas/Medicamento/FET/2010/ FET+numero+3+Agomelatina.htm

(44) Scholdemayer WS. Public citizen update: competition and pricing issues in the pharmaceutical Market. Prime Institute, University of Minnesota, 2000.

(45) Soger A, Socolar D, Pharmaceutical marketing and research spending: the evidence does not support PhRMA`s claims, presentado en: American Public Health Association Annual Meeting, 2001, Atlanta, Georgia, disponible en: http://dcc2.bumc.bu.edu/hs/ushealthreform.htm.

(46) Govern de les Illes Balears, Quetiapina de liberación prolongada, Informe de Evaluación de Medicamento, $\mathrm{n}^{\circ} 10,2009$. ISSN: 1989-192X.

(47) Servicio Madrileño de Salud, Estereoisómeros ¿Aportan algo en terapéutica?, Notas Farmacoterapéuticas, SaludMadrid vol. 15 nº10, 2008, disponible en: http://www.madrid.org

(48) Servicio Navarro de Salud, Paliperidona, Ficha de Evaluación Terapéutica nº4, 2008, disponible en: http://www.navarra.es/home_es/Temas/Portal+de+la+ Salud/Profesionales/ Documentacion+y+publicaciones/Publicaciones+tematicas/Medicamento/FET/2008/FET+2008+4.htm

(49) Servicio Canario de la Salud, Prescripción prioritaria por principio activo de las 7 moléculas con mayor impacto económico, disponible en: http://www2. gobiernodecanarias.org/sanidad/scs/content/ ea31c953-5830-11df-8125-5700e6e02e85/PrescripcionPrioritariaPA_7Moleculas.pdf

(50) Servicio Canario de la Salud, Genéricos y Bioequivalencia: ¿seguimos dudando?, Infarma, 2010, vol.2, n², disponible en: http://www2.gobiernode canarias.org/sanidad/scs/content/e5780b9f-61a211df-bf4e-0946409fd556/ Infarma_Genericos.pdf 
ORIGINALES Y REVISIONES

(51) Servicio de Farmacia Hospital Universitario 12 de Octubre, Madrid, Medicamentos genéricos: ¿qué significa eso del +/- 20\%?, Actualidad del medicamento, 2007, nº5.

(52) Pearce GA, McLachlan AS, Ramzan I, Bioequivalence: how, why, and what does it really mean?, J Pharm Pract Res, 2004; 34: 195-200.

(53) La OMS ocultó que sus expertos en gripe A cobraron de farmacéuticas, El País, 5 de junio de 2010. 